Retention of English sentences learned by reading aloud using Text-To-Speech (TTS) speech sounds: A longitudinal study in a Japanese high school

Kataoka, Harumi $₫$

KinkiUniversity, Japan (h.kataoka2014@gmail.com; h_kataoka@kindai.ac.jp)

Ito, Makiko

Osaka Prefectural Kashiwara-higashi High School, Japan (itou@kashiwarahigashi.osaka-c.ed.jp)

Yamane, Shigeru

Kansai University, Japan (yamane@kansai-u.ac.jp)

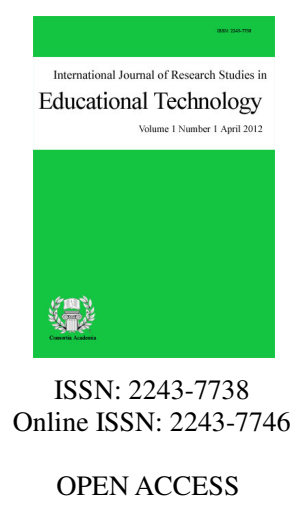

OPEN ACCESS

\title{
Abstract
}

This study examines the retention effect of classroom English sentences, which Japanese high school students learned through the activity of reading aloud (RA). The audio materials used during their instruction were created by using Text-To-Speech (TTS) speech sounds. From 2009 to 2011, we conducted a longitudinal study spanning three academic years at a public senior high school in Japan. In total, 49 Japanese high school students participated in this study. They were divided into two groups: (a) an RA instruction group, and (b) a non-instruction group. Following the methods of experiments in certain previous studies on RA, cloze tests were given to students in order to monitor their memory transition. The tests were conducted three times, as follows: (1) in April 2009 when they had entered the high school, (2) in June 2009 when the RA instruction group had learned 20 classroom English sentences over the course of five lessons, and (3) in December 2011 when they were the third grade students. The results of the retention rate showed that the students in the RA instruction group sustained a higher retention than those in the non-instruction group. This result and comments by students in the RA group indicate that the scaffolding by a teacher during lessons had long-term effects on students' abilities in retaining classroom English sentences.

Keywords: classroom English sentences; reading aloud; TTS speech sounds; memory; English as a foreign language education 


\section{Retention of English sentences learned by reading aloud using Text-To-Speech (TTS) speech sounds: A longitudinal study in a Japanese high school}

\section{Introduction}

The Ministry of Education, Culture, Sports, Science and Technology (MEXT) of the Japanese government published a new curriculum stipulating that English (not Japanese) should be used as the main language of instruction during high school English lessons beginning in the academic year of 2013 (MEXT, 2009, p. 92). Thus, Japanese high school students are now required to learn and memorize basic classroom English in order to understand what their teachers say in class.

Although several books on classroom English are available in Japan (Abe, 2001; Hughes, 1981; Someya, 1993), they are no more than a list of expressions of basic classroom English conversation. There are not enough academic studies of high school students learning classroom English in class.

Reading aloud (RA), called ondoku in Japanese, is widely used in Japan as an English language teaching method. The results of previous studies on RA (Ishihara, 2002; Miyasako, 2002; Suzuki, 1998; Takeuchi, Ikeda, \& Mizumoto, 2012) have shown that RA is an effective method for Japanese learners to acquire English. However, to the best of our knowledge, no study has been conducted on learning classroom English by RA in a Japanese high school class. Thus, further research on the effectiveness of RA activity, and the amount of classroom English that high school students retain in their memory is necessary.

MEXT (2010) recommended using information and communication technology (ICT) in high school English education. Text-To-Speech (TTS) synthesis technology is a form of ICT. By employing the TTS system, teachers can digitally create English audio materials suitable for their students' English proficiency, and they can use them during English lessons (Kataoka \& Ito, 2013). TTS technology is already widely used in our daily lives (Kataoka, 2009; Azuma, 2008), and it is also applied as a speech-recognition system and/or as an automated voice-answering system in the latest smartphones (as of 2015).

However, there are not enough studies using TTS speech sounds in Japanese high school English education. Therefore, we investigated the effectiveness of RA activity by using TTS speech sounds to examine students learning 20 classroom English sentences in a Japanese high school.

\section{Literature Review}

\subsection{Language Recognition}

English language education in Japanese high schools is generally conducted within the scope of the curriculum guidelines set by the MEXT. The curriculum guidelines aim to cultivate students' four language skills, that is, listening, speaking, reading, and writing, through their English lessons (MEXT, 2009, pp. 87-92).

It has generally been agreed by English language teachers that the balanced development of learners' four language skills contributes to their understanding of the target language. Jones (1918/1976, p. 61), for instance, stated that "those learning to speak a foreign language should begin their study by ear-training, which will enable them to recognize the sound of language." According to Widdowson (1978, p. 57), the four language skills are closely interrelated. He explained that learners of English as a Foreign Language (EFL) need to learn (a) spelling, (b) speech sounds, and (c) the meaning of each lexical item, which would be the first step to understanding English. 
In Japanese EFL education, Palmer (1921/1999, pp. 400-401), who had worked on constructing basic English education in modern Japan and was an assistant to Daniel Jones at University College London (Tamagawa Academy and University, 2014), indicated that enhancing Japanese students' listening abilities to understand English speech sounds is the basis of English language learning. Hatori (1977, p. 74) stated that it is important for Japanese EFL learners to learn and understand both the spelling and the speech sounds of English words. He also insisted that Japanese students' motivation in learning would be promoted by presenting audio materials to learners in class. These studies imply the importance of listening activity in acquiring English, which has different phonological structures from Japanese. These previous studies have stated the importance of developing Japanese students' listening abilities. Re-examining the validity of using audio materials during English lessons in Japanese schools is necessary.

A human cognitive model was defined by Kohno (1984) as a process in which visual and audio information is recorded in the brain, and thus, the information obtained through these two channels interacts to retain it as a permanent memory. For language recognition, he stated that memorizing each lexical item and its pronunciation on a sequential basis is important for Japanese students. Based on this cognitive model, various studies have been conducted on how Japanese EFL learners recognize English visual and audio information (Kohno, Ikari, Ishikawa, Kadota, Murata, \& Yamane, 2007).

These previous studies covered in this section indicate that understanding English speech sounds is a basis in developing English proficiency for Japanese EFL learners, resulting in correct language recognition. As previous studies suggest, acquiring a listening ability helps develop learners' four language skills. Thus, it is important for Japanese high school students to listen to English as much as they can.

\subsection{RA Studies in Japan}

In RA activity, learners read English sentences silently while listening to English speech sounds. They then say them aloud, while looking at them. During RA activity, therefore, students are expected to practice three of the four language skills (i.e., reading, listening, and speaking).

Suzuki (1998) conducted a comparative study on RA in a high school. The results of the English Test in the National Center for University Entrance Examinations in Japan revealed that students who had participated in RA obtained higher test scores than those without RA in class. These results showed that RA during classroom lessons helped improve Japanese high school students' English proficiency.

Ishihara (2002) also conducted a study on RA, with 80 second graders of a Japanese high school as participants. They were separated into two groups (i.e., students in one group learned with RA, whereas those in other group did not). The results of a cloze test showed that 40 students in the RA group obtained higher scores compared to the 40 students in the non-RA group. The results suggest that the RA activity worked effectively in having the students retain the text they had read in their memory. The RA activity was researched by Miyasako (2002). The participants in this study were 40 high school students in Japan. The results revealed that this activity developed students' English grammar, vocabulary, and listening and reading abilities, suggesting that RA could help enhance high school students' English proficiency.

Although RA has been reported to be an effective English learning method for Japanese EFL learners, not many studies have analyzed it medically. To fill this gap, Takeuchi et al. (2012) investigated Japanese EFL learners' RA activity by examining cerebral activation. They observed a higher degree of cerebral activation while the participants were engaged in RA in their L2 (English) than in their L1 (Japanese). The results of these previous studies imply that RA is an effective way for Japanese EFL learners to study English. Although RA studies on Japanese high school students are available, experimental period of each study was within a year. The studies that have focused on retention in long-term memory over a longer experimental period are yet to be conducted. We wanted to examine how RA functions in helping students retain texts in their memory over a longer experimental period. 


\subsection{Memory}

Epoch-making experiments on human memory were conducted by Ebbinghaus (1885/1998). The results showed that the accuracy rate of memory retrieval was $58.2 \%$ after 20 minutes of the experiment, but that the rate plunged to $21.1 \%$ after 31 days of learning. His results suggest how human memory is temporal and easily fades. Kataoka (2007) conducted research on the memory of Japanese high school students. The participants were 102 third-year high school students, who were asked to learn five English and Japanese proverbs. The results revealed that the correct retention rate of English proverbs was $66.19 \%$, whereas that of Japanese proverbs was $95.83 \%$ after 51 days of learning. These results showed that it is more difficult for Japanese high school students to retain information correctly in their memory in L2 (English) than in L1 (Japanese). It was reported by Tinkham (1993) that most learners require five to seven repetitions to memorize each lexical item in English. Webb (2007) researched the relation between repetition time and memory. The participants in his experiment were 121 Japanese university students. The results revealed that 10 repetitions were optimal for longer memory retention.

These previous studies have indicated that using English speech sounds during lessons is an effective learning strategy for Japanese EFL learners to master English. However, the number of relevant studies conducted for high school students learning English is not enough (inadequate). Therefore, we felt it necessary to conduct a further study on learning English in Japanese high school settings.

\subsection{TTS Studies in EFL Education}

Because of recent advancements in computer technology, English language teachers can create digital audio materials suitable for their students' English proficiency level by using a TTS system on their computers. Several studies on TTS technology in EFL education have already been conducted in Japan as well as overseas. In Australia, for instance, Jones, Berry, and Stevens (2007) conducted a comparative study on the effect of TTS speech sounds and human voice. Their results revealed that no perception gap exists between a native English speaker's voice and TTS speech sounds when participants listened to them. The results implied the plausibility of using TTS speech sounds in English education. Chew (2009) introduced the methods of using TTS technology to produce English language teaching materials in Singapore.

In Japan, Azuma (2008) reported on the introduction of TTS technology to (a) a CALL system, and (b) in a Moodle-based e-learning environment at a Japanese university. Yoshida (2008) conducted a study of an electronic phrasal verb workbook using GIF animation combined with TTS speech sounds at a Japanese university. His result revealed that the moving images combined with TTS speech sounds were effective for Japanese university students learning English. Kataoka (2009) tested three types of speech sounds: (a) TTS speech sounds, (b) a British speaker's voice, and (c) an American speaker's voice. The participants were Japanese undergraduate and graduate students. The results revealed that the participants did not show any perception gap among the three types of speech sounds, indicating that TTS speech sounds can be used as audio English language learning materials for Japanese EFL learners. Hirai and O'ki (2011) examined the quality of TTS speech sounds and native speakers' voice. The participants in their experiment were Japanese university students who were divided into groups based on their English proficiency. The results of the listening tests showed that TTS speech sounds were perceived to be almost as natural as a native speaker's voice by the students in both the upper- and lower-proficiency groups. In addition, the students in the lower-proficiency group tended to prefer TTS speech sounds to the native speaker's voice. A study was conducted by Kataoka and Ito (2013) on RA instructions by TTS speech sounds and a Japanese teacher's voice. In total, 151 male and female first-year Japanese high school students participated in that study. They were given review lessons on basic English verbs by RA instruction using two types of speech sounds. The results showed that TTS speech sounds used in their study had had sufficient usability as speech sounds for Japanese high school EFL education.

The results of these previous TTS studies have shown that TTS speech sounds had sufficient usability as 
speech sounds for English language education in Japan. Using TTS speech sounds as audio materials has four major advantages over using a native speaker's voice in Japanese EFL education (Kataoka, 2009; Azuma, 2008), as follows:

$>\quad$ By using a TTS audio database downloaded onto teachers' computers, teachers can produce English audio materials on their own. They do not need to ask for assistance from native speakers.

$>\quad$ Teachers do not have to be concerned with additional finances, which may be necessary when asking native speakers to perform a recording of their voice.

$>\quad$ Because the TTS speech sounds can control the speed and/or pause during speech sounds, teachers can create audio materials that match their students' English proficiency.

$>\quad$ The TTS speech sounds can be used to synthesize a native speaker's voice that was originally recorded onto a computer; therefore, stable speech sounds can be created.

Considering these four advantages, TTS speech sounds seem to be useful for both Japanese EFL learners to acquire English and for English language teachers to create audio language learning materials that match their students' English proficiency. However, the number of research results on the actual use of TTS speech sounds as English audio materials for Japanese high school EFL education is not enough.

\section{Study: Retention of Classroom English Sentences}

\subsection{Objective}

Previous studies have indicated that RA (Ishihara, 2002; Miyasako, 2002; Suzuki, 1998; Takeuchi et al., 2012) and learning by repetition (Tinkham, 1993; Webb, 2007) are efficient methods for Japanese EFL learners to learn English. Although various types of research have been conducted on RA, no study has investigated learning classroom English sentences with RA in Japanese high school English lessons. A previous study conducted by Kataoka and Ito (2013) on the effectiveness of TTS speech sounds in a senior high school revealed that TTS speech sounds are of sufficient quality to be used as English listening materials. However, there are not enough research results on how TTS speech sounds can be used during high school English lessons in Japan. Thus, this study investigates the effectiveness of RA activity with TTS speech sounds, and the amount of classroom English the high school students retain in their memory after listening, reading, and repeating activities. For this longitudinal study, we compared the retention of classroom English sentences between an RA instruction group and a non-instruction group.

\subsection{Twenty Classroom English Sentences}

By referring to several previous classroom English books (Abe, 2001; Hughes, 1981; Someya, 1993), we created 20 classroom English sentences to be used in this study. These sentences are considered common classroom English phrases that are frequently used in Japanese high school English classes (see Appendix A).

\subsection{TTS Speech Sounds Used in This Study}

Dutoit (1997) stated that TTS systems are composed of two items: (a) a TTS engine, and (b) a/some TTS audio database(s). Taylor (2009, p. 2) discusses the applicability of TTS in our daily lives. We hope that many English teachers in Japan can create their original audio materials with TTS that matches their students' level of English proficiency. With this in mind, we chose a more economical approach to create the TTS system, which we already used in our earlier experiment to investigate the effectiveness of TTS in a Japanese high school (Kataoka \& Ito, 2013). The results revealed that it was of sufficient quality to be used as English audio materials for Japanese high school EFL learners. The free TTS software application "Balabolka Ver. 1.9.0.241," which enabled us to define the duration of a short pause by up to 1/1000 of a second, was used as a TTS engine in this study. For the TTS audio database ${ }^{1}$ we installed a male and a female TTS voice, both in American English, on a 
Kataoka, H., Ito, M., \& Yamane, S.

personal computer (Kataoka, 2009). Figure 1 shows a screenshot of the TTS system used in this study.

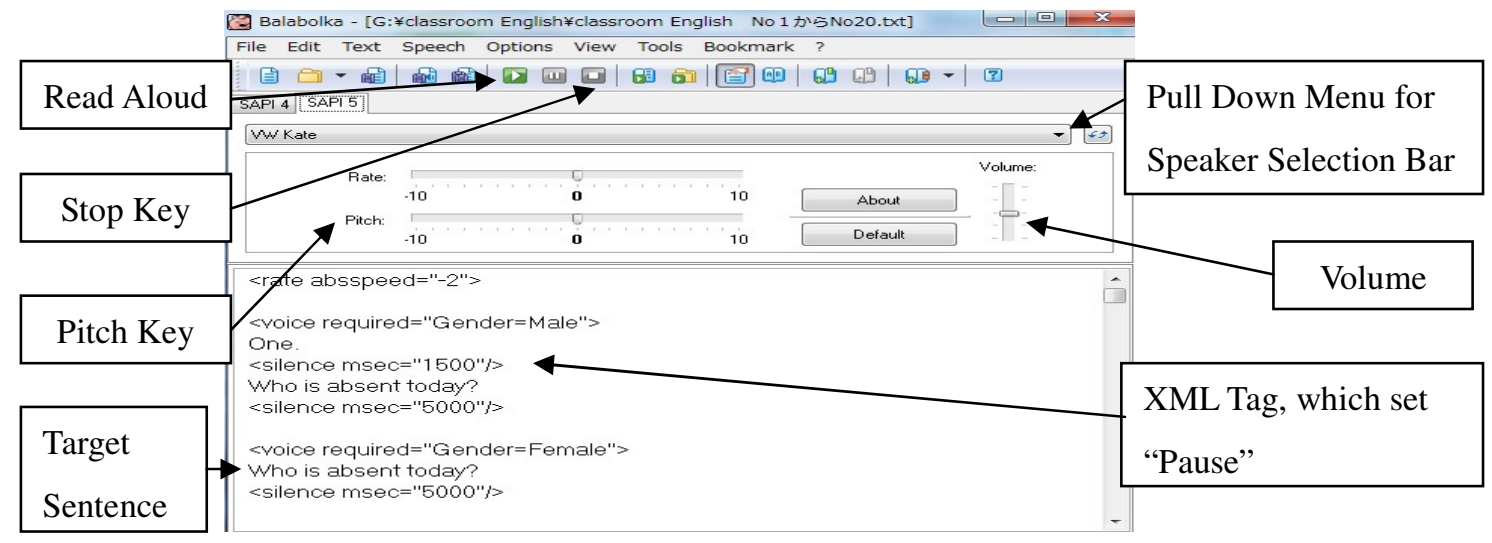

Figure 1. A Screenshot of the TTS System

The classroom English audio used in this study was created using this TTS system, and was recorded onto a $\mathrm{CD}$. The $\mathrm{CD}$ was played using a $\mathrm{CD}$ deck. Because of the fact that the regular classrooms used in this study were too old to use a computer, we decided to use the CD deck instead of the computer.

\section{Experiment 1: Speech Analyses}

There are not enough acoustic research results on audio quality of TTS speech sounds. This may be one of the reasons why some native and Japanese English teachers in Japan take a negative stance toward using TTS speech sounds for their lessons. In order to investigate the audio quality, we conducted the following two types of speech for the CD analysis: (a) acoustic analysis using the speech analyzer "Praat," and (b) speech evaluation conducted by raters.

\subsection{Results of Acoustic Analyses using the Speech Analyzer "Praat"}

Two types of speech sounds were used in this study, as follows: (a) a male TTS voice, "Paul 16," and (b) a female TTS voice, "Kate 16." The speech sounds were analyzed using Praat software. Praat is a free software program that is widely used to analyze speech sounds. For example, Halliday and Greaves (2008) present the results of acoustic analyses by Praat, and recommend its use in analyzing and examining speech sound features. In Japan, Komatsu (2011, p. 120) also recommends Praat as a speech analyzer.

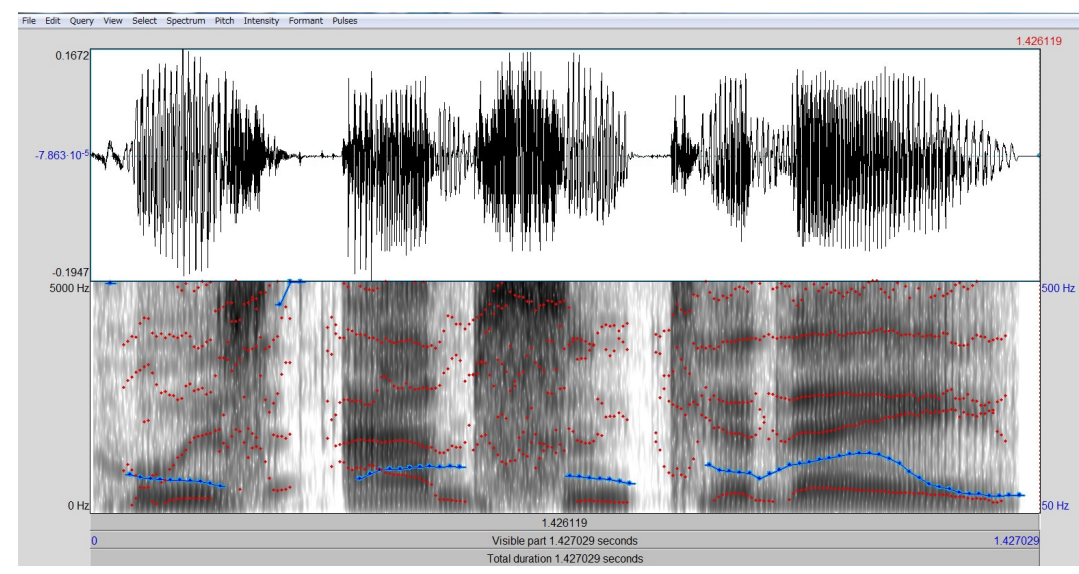

Figure 2. A Screenshot of the Speech Analyzer "Praat"

Ladefoged (2003, p. 75) states that pitch can be a useful parameter in examining the features of speech sounds. To identify the features of the two voices, we measured their duration, maximum and minimum pitch, and pitch range. We analyzed each speech sound in the range from $50 \mathrm{~Hz}$ to $500 \mathrm{~Hz}$, referring to the definition of 
speech analyses (Halliday \& Greaves, 2008, p. 30). Table 1 shows the mean figures of these variables belonging to the two types of speech sounds used in this study.

\section{Table 1}

Mean Figures of 20 English Sentences in Two Types of Speech Sounds

\begin{tabular}{lcccc}
\hline & & \multicolumn{3}{c}{ Pitch (Hz) } \\
\cline { 3 - 4 } Speech Sounds & Duration (s) & Max F0 & Mini F0 & Range \\
\hline TTS Male & 1.28 & 246.53 & 83.62 & 162.91 \\
TTS Female & 1.36 & 260.11 & 132.45 & 127.66 \\
\hline
\end{tabular}

Note. "s" represents second.

As expected, both the maximum pitch and the minimum pitch in the female TTS speech sounds were higher than those in the male speech sounds (see Table 1). The duration of female speech lasted longer than the male speech. The data clearly showed that the TTS female speech sounds had a higher pitch, and were slower than the TTS male speech sounds.

\subsection{Results of Speech Evaluation by Raters}

Seven English teachers participated in this study as raters. They were six Japanese English teachers and one British English teacher. They were all employed at the Japanese high school in which we conducted the experiment for our study. They were asked to listen to the two types of speech sounds on the CD (see section 3.3), and then they evaluated them based on the CD's intelligibility, comprehensibility, and naturalness as spoken English on a five-point Likert scale (Takeuchi, 2003, p. 254). The results are shown in Table 2. The $\alpha$ values of the two types of speech sounds were over $0.70,{ }^{2}$ and revealed no significant difference among the seven raters' judgments.

Table 2

Results of the Two Types of Speech Sounds as Evaluated by Seven Raters

\begin{tabular}{lccc}
\hline \multicolumn{1}{c}{ Speech Sounds } & $M$ & $S D$ & $\alpha$ \\
\hline TTS Male & 3.796 & 0.863 & 0.922 \\
TTS Female & 3.510 & 0.814 & 0.862 \\
\hline
\end{tabular}

Note. Cronbach's coefficient alpha, signified as " $\alpha$," shows the inter-rater reliability.

Table 2 shows that the TTS male speech sounds obtained a higher mean score than the TTS female speech sounds. In order to compare the two types of speech sounds, we conducted a two-tailed independent $t$ test. ${ }^{3}$ The result showed that a statistically significant difference did not exist between the two types of speech sounds $(t=$ $0.848, d f=14, p=.413, d=0.341$ ), and the effect size was medium. These results show that although the raters felt the TTS male speech to be more intelligible, comprehensible, and natural as English compared to the female speech, the perceived difference was not statistically significant.

\section{Experiment 2: Classroom English Sentences Learning by RA}

\subsection{Participants}

In total, 49 Japanese public senior high school students participated in our study. They entered a high school in April 2009, and graduated in March 2012. In order to explore the effect of RA using TTS speech sounds, they were divided into the following two groups based on their regular classes: (a) the RA instruction group $(n=20,9$ male students and 11 female students), and (b) the non-instruction group ( $n=29,14$ male students and 15 female students). They were all born in Japan, and had been studying English for more than three years. None of them had stayed in an English-speaking country, or had any experience of participating in this type of experiment. We 
Kataoka, H., Ito, M., \& Yamane, S.

ensured that the participants remained anonymous throughout the study. Their English proficiency level was not high; the participants' academic record, called naishinten in Japanese, which had been submitted when they took their high school entrance examination, was 4.2 points on a scale ranging from 0 to 10 . They were beginner-level EFL learners.

\subsection{The Study Context}

Before introducing this experiment, the study context is elaborated as follows: We conducted this study in a public high school. To investigate the memory span and the accuracy rate, we performed this research project from 2009 to $2011^{4}$. To compare the changes in the correct retention rate of what they had learned, we divided the research years into the following three parts: (1) in April 2009 when the participants had entered the high school, (2) in June 2009 when the students in the RA instruction group had learned 20 sentences of classroom English over the course of five lessons, and (3) in December 2011 when all participants were in their third year of high school. Author 2 taught English to all the participants in this study.

\subsection{Research Questions}

Because the students in the RA instruction group learned 20 classroom English sentences in class, we expected that they would keep a higher accuracy rate than the students in the non-instruction group. However, it was necessary to validate the difference in memory retention between the two groups.

Our Research Questions are as follows:

$>$ How much difference, if any, in accurate retention rate exists between the RA instruction group and the non-instruction group?

$>\quad$ What do the students in the RA instruction group think about the RA activity?

\subsection{Research Design and Procedure}

To investigate the learning effect and memory retention of RA instruction using TTS speech sounds to help Japanese high school students learn classroom English, all of our study participants were divided into the following two groups based on their regular classes: (a) the RA instruction group, and (b) the non-instruction group. Following the steps graphically illustrated in Figure 3, tests were administered to the participants.

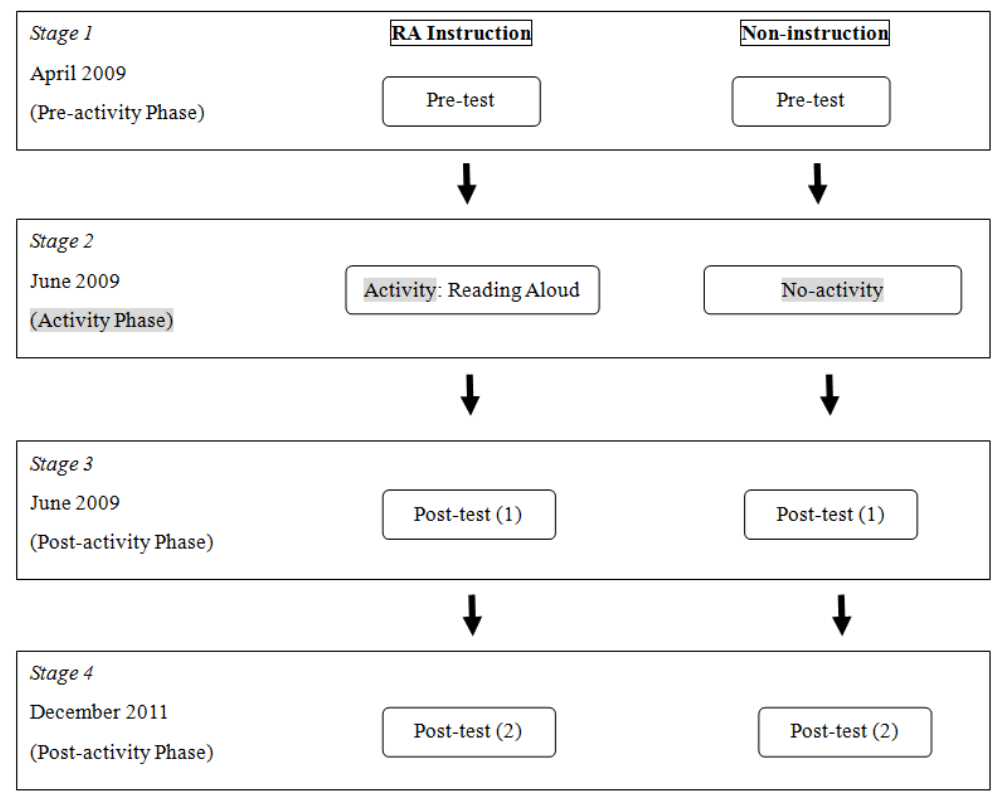

Figure 3. Procedure of the Experiment 2 


\subsection{Procedure in RA Instruction Group}

Table 3 lists the four stages of the learning process, which were adopted for the RA instruction group in our study.

\section{Table 3}

Timeline for RA Instruction Group in This Study

\begin{tabular}{|c|c|c|c|c|c|}
\hline & Day & Activities & Audio Instruction & English Sentences & Time Frame \\
\hline \multicolumn{6}{|l|}{ Stage 1} \\
\hline April 2009 & 1 & Pre Test & No Audio Instruction & No. 1 - No. 20 & $7.00 \mathrm{~min}$. \\
\hline \multicolumn{6}{|l|}{ (Pre-activity Phase) } \\
\hline \multicolumn{6}{|l|}{ Stage 2} \\
\hline June 2009 & 2 & Activity Sheet (1) & TTS CD Sounds & No. 1 - No. 5 & $1.15 \mathrm{~min}$. \\
\hline \multirow[t]{4}{*}{ (Activity Phase) } & 3 & Activity Sheet (2) & TTS CD Sounds & No. 6 - No. 10 & $1.10 \mathrm{~min}$. \\
\hline & 4 & Activity Sheet (3) & TTS CD Sounds & No. 11 - No. 15 & $1.14 \mathrm{~min}$. \\
\hline & 5 & Activity Sheet (4) & TTS CD Sounds & No. 16 - No. 20 & $1.18 \mathrm{~min}$ \\
\hline & 6 & Activity Sheet (1) - (4) & TTS CD Sounds & No. 1 - No. 20 & $4.57 \mathrm{~min}$ \\
\hline \multicolumn{6}{|l|}{ Stage 3} \\
\hline June 2009 & 7 & Post Test (1) & No Audio Instruction & No. 1 - No. 20 & $7.00 \mathrm{~min}$. \\
\hline \multicolumn{6}{|l|}{ (Post-activity Phase) } \\
\hline \multicolumn{6}{|l|}{ Stage 4} \\
\hline December 2011 & 8 & Post Test (2) & No Audio Instruction & No. 1 - No. 20 & $7.00 \mathrm{~min}$. \\
\hline (Post-activity Phase) & & & & & \\
\hline
\end{tabular}

Considering the number of regular high school English lessons and learning content stipulated by MEXT, we decided to use five lessons in order to teach classroom English with RA instruction (see Table 3). We used 20 classroom English sentences for this study (see Appendix A). After carefully considering the students' English proficiency level (see section 5.1) and their regular academic performance, we decided to teach five classroom English sentences per activity sheet (see Appendix B) from Day 2 to Day 5 (see Table 3).

According to certain previous studies (Hatori, 1977; Kohno, 1984; Palmer, 1999 [1921]), knowing both the pronunciation and meaning of each English word and its Japanese equivalent would facilitate Japanese high school students' understanding. We also found that our study participants had to be presented equivalent sentences in Japanese for them to understand the meaning of each classroom English sentence. Therefore, five sentences of classroom English and their Japanese translations were handed to the students as an activity sheet (see Appendix B). Day 6 was the last day of RA instruction, when the students reviewed the 20 classroom English sentences (see Table 3).

The classroom English audio was created using the TTS system, and recorded onto a CD (see section 3.3). The TTS CD was played using a CD deck, and given to the participants of this study. Pauses of 5,000 ms (i.e., 5 s) were inserted between sentences, so that the students could repeat them together in class. In order to exclude the gender influence in the speech stimuli, each sentence in both the male and female TTS speech sounds was provided to the students, so that they could repeat one classroom English sentence twice in one lesson (see Table 4). 
Kataoka, H., Ito, M., \& Yamane, S.

\section{Table 4}

Contents of Audio Materials

\begin{tabular}{llll}
\hline Classroom English No. & Texts & TTS Sounds & Pause (s) \\
\hline 1 & One. & Male & 1.50 \\
Who is absent today? & Male & 5.00 \\
& Who is absent today? & Female & 5.00 \\
\hline
\end{tabular}

The following is a description of the two-step learning process adopted for RA instruction (see Figure 4):

(1) The first step was a "reading and listening" stage. Students were given an activity sheet (see Appendix B), which they read quietly while listening to TTS speech sounds.

(2) The second step was a "repeating" stage. After the first step, all the students repeated aloud in unison each classroom English sentence exactly as they had heard it, while looking at each sentence on the activity sheet.

Step 1: Reading and Listening Stage

\begin{tabular}{|c|c|}
\hline Listening: Quietly & Reading: Quietly \\
\hline $\begin{array}{c}\text { TTS } \\
\text { Sounds }\end{array}$ & $\begin{array}{c}\text { Activity } \\
\text { Sheet }\end{array}$ \\
\hline
\end{tabular}

Step 2: Repeating Stage



\begin{tabular}{|c|c|}
\hline No listening & Reading: Actively \\
\hline Activity \\
Sheet
\end{tabular}

Figure 4. Two-step Learning Process in RA Instruction

This RA task was conducted for 1.10 to 4.57 minutes during the five lessons (see Table 3 ). The students listened to the audio twice for every sentence (see Table 4), with a total repetition time of four.

\subsection{Procedure in the Non-Instruction Group}

All students in the non-instruction group were handed a list of 20 classroom English sentences (see Appendix C), and asked to study them on their own at home.

\subsection{Cloze Test}

We selected the 20 classroom English sentences from previously published books on classroom English (Abe, 2001; Hughes, 1981; Someya, 1993), and rewrote them for this study (see Appendix A). We decided to use cloze tests to monitor the progress of learners' English proficiency by referring to previous RA studies (Ishihara, 2002; Miyasako, 2002; Suzuki 1998). In order to monitor the students' memory transition, we used the same 20-item cloze tests (see Appendix D) for the pretest and two posttests. The cloze test in this study comprised multiple-choice questions. Students were asked to select the best answer in order to complete each sentence, and then write a number (i.e., 1, 2, 3 or 4) on their cloze tests (see Appendix D). One point was given 
to each correct answer, for a full score of 20. This type of cloze test with four multiple-choice options per question is often used in university entrance examinations in Japan, as (a) the first-stage English Test of the National Center for University Entrance Examinations, and as (b) English tests in many private universities. We considered that this type of test should be given to Japanese high school students as much as possible in order to acquaint them with this style. This is the reason we used the cloze test with four multiple-choice options for this study.

To facilitate students' understanding of classroom English, both English and Japanese sentences were printed on the activity sheet (see Appendix B), the list of classroom English (see Appendix C), and also on the cloze test (see Appendix D).

\subsection{Basic Data of Two Groups}

Prior to the experiment, we administered a 20-item cloze test (see Appendix D) to two groups, the RA instruction group and the non-instruction group, to measure their English proficiency. For each correct answer, one point was given, for a maximum possible score of 20 . The preliminary data are summarized in Table 5.

\section{Table 5}

Basic Data of Two Groups

\begin{tabular}{lcccccc}
\hline & & & & & \multicolumn{2}{c}{$95 \%$ CI } \\
\cline { 5 - 7 } Group & $n$ & $M$ & $S D$ & $S E$ & $L L$ & $U L$ \\
\hline RA Instruction & 20 & 10.800 & 1.795 & .401 & 9.960 & 11.640 \\
Non-instruction & 29 & 11.310 & 1.834 & .341 & 10.613 & 12.008 \\
\hline
\end{tabular}

Note. The full score of the cloze test is 20 .

A two-tailed independent $t$ test confirmed that a statistically significant difference did not exist between the scores of the two groups $(t=-0.966, d f=47, p=.339, d=0.280)$, and the effect size was medium. The result of a Shapiro-Wilk test for the RA instruction group was 0.064 , and that for the non-instruction group was 0.199 , indicating that the scores of both student groups showed a normal distribution. The students in both groups, therefore, gained equivalent scores on the 20-item cloze test. Thus, the English proficiency level of the two groups was considered homogeneous at the outset of the experiment.

\section{Results and Discussion}

\subsection{Results of the Cloze Tests}

As mentioned earlier, the same cloze tests were administered to the participants in order to monitor their learning process (see section 5.4). Table 6 presents in summary form the results of the cloze tests.

Table 6

Results of the Cloze Tests

\begin{tabular}{|c|c|c|c|c|c|c|c|}
\hline \multirow[b]{3}{*}{ Group } & \multirow[b]{3}{*}{$n$} & \multicolumn{6}{|c|}{ Time } \\
\hline & & \multicolumn{2}{|c|}{ April 2009} & \multicolumn{2}{|c|}{ June 2009} & \multicolumn{2}{|c|}{ December 2011} \\
\hline & & $M(S D)$ & $95 \% \mathrm{CI}$ & $M(S D)$ & $95 \% \mathrm{CI}$ & $M(S D)$ & $95 \% \mathrm{CI}$ \\
\hline RA Instruction & 20 & $10.800(1.795)$ & {$[9.960,11.640]$} & $13.900(2.654)$ & {$[12.658,15.142]$} & $12.600(3.202)$ & {$[11.101,14.099]$} \\
\hline Non-instruction & 29 & $11.310(1.834)$ & {$[10.613,12.008]$} & $11.000(2.188)$ & {$[10.168,11.832]$} & $10.414(3.333)$ & {$[9.146,11.682]$} \\
\hline
\end{tabular}

Note. One point was given for each correct answer, with a total score of 20. 
Kataoka, H., Ito, M., \& Yamane, S.

Figure 5 shows the score fluctuations over the three test times in both groups.

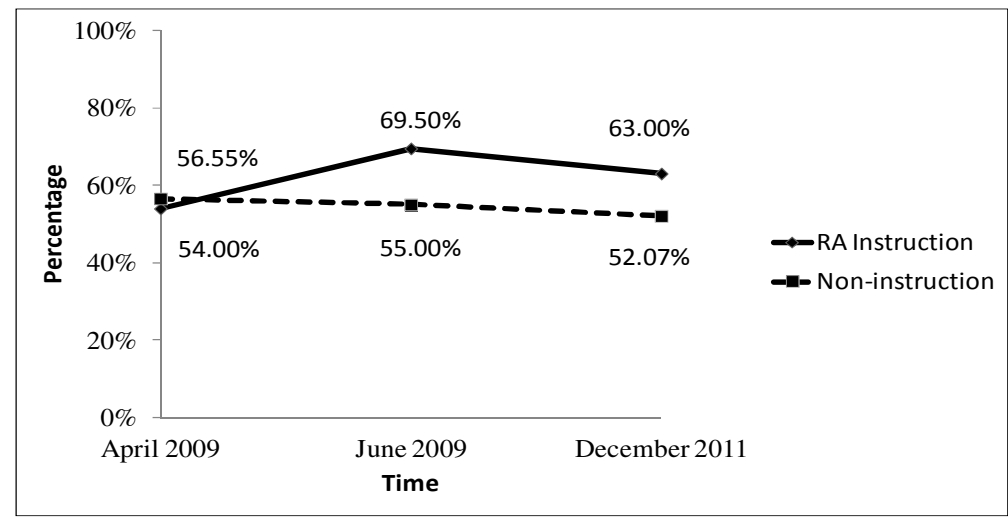

Figure 5. Accuracy Rates of the Cloze Tests

The results of the cloze tests (see Table 6 and Figure 5) show that the highest correct retention rate was obtained in June 2009 by the RA instruction group, and a higher retention rate by the same group was sustained until December 2011, when all the participants became third-year high school students.

These results show that the students in the RA instruction group had retained in their memory what they had learned during the five RA lessons with a higher accuracy rate compared to the non-instruction group, for 2.5 years.

\subsection{Analyses of Two-way ANOVA}

We analyzed the results of the cloze tests (see Table 6 and Figure 5) by using a $2 \times 3$ (Group $\times$ Time) design of two-way repeated-measures ANOVA. The analysis was conducted using SPSS 19.0 for Windows. Statistical significance was set at the .05 level for all analyses. Significant differences were detected on the main effect of time $\left[F(2,94)=4.464, p=.014\right.$, partial $\left.\eta^{2}=.087\right]$, and on the main effect of the group $[F(1,47)=9.158, p$ $=.004$, partial $\eta^{2}=.163$ ], as well as on the interaction between the two variables [i.e., time and group; $F(2,94)$ $=7.127, p=.001$, partial $\left.\eta^{2}=.132\right]$. After considering these results, we chose to apply within-subject and between-subject designs of two-way ANOVA to our data.

The data was analyzed using a within-subject design of two-way ANOVA. The analysis of the simple main effect test (variable: time) showed a significant difference in the RA instruction group $[F(2,94)=9.020, p$ $=.000]$, but no significant difference in the non-instruction group $[F(2,94)=1.120, p=.331]$. The results of cross-comparison are shown in Table 7.

\section{Table 7}

Results of Cross-comparison: Time

\begin{tabular}{lcc}
\hline & \multicolumn{2}{c}{ Sig. of $F$} \\
\cline { 2 - 3 } \multicolumn{1}{c}{ Comparison } & RA Instruction & Non-instruction \\
\hline April 2009 vs. June 2009 & $.001^{*}$ & .535 \\
April 2009 vs. December 2011 & $.038^{*}$ & .207 \\
June 2009 vs. December 2011 & .084 & .342 \\
\hline
\end{tabular}

Note. The analysis was conducted using Bonferroni correction.

In the RA instruction group, there were significant differences in April 2009 versus June 2009, and in April 2009 versus December 2011, whereas no significant difference was detected for June 2009 versus December 2011. In the non-instruction group, there was no significant difference for the three testing times. These results show that RA activity in June 2009 proved the effectiveness of retention for the students regarding the 20 
classroom English sentences.

Next, we analyzed the data by using a between-subject design of two-way ANOVA. The results of the simple main effect test (variable: group) are shown in Table 8.

Table 8

Results of Simple Effect: Group

\begin{tabular}{lccc}
\hline & \multicolumn{3}{c}{ Sig. of $F$} \\
\cline { 2 - 4 } Comparison & April, 2009 & June, 2009 & December, 2011 \\
\hline RA Instruction vs. Non-instruction & .339 & $.000^{*}$ & $.026^{*}$ \\
\hline
\end{tabular}

There were significant differences between the two groups in June 2009 and December 2011, whereas no significant difference was found in April 2009. These results and Figure 5 show that the RA instruction group had been retaining a higher learning effect than non-RA groups during June 2009 to December 2011.

Research Question 1 asked the following: "How much difference, if any, in accuracy retention rate exists between the RA instruction group and the non-instruction group?"

In summary, it became clear from our longitudinal experiment that the RA instruction group, after receiving the RA instruction in June 2009, had sustained a higher retention rate and learning effect compared to the non-instruction group until December 2011, that is, for 2.5 years.

\subsection{Comments by Students in the RA Instruction Group}

Research Question 2 asked the following: "What do the students in the RA instruction group think about the RA activity?" We wanted to know how much the students in the RA group remembered after using a TTS audio CD when they had studied 20 classroom English sentences in June 2009, and what they felt toward classroom English itself. This is the reason we chose to conduct a questionnaire with the post-cloze test in December 2011.

A questionnaire sheet was given to each student in the RA instruction group in December 2011. The questionnaire asked two questions, as follows:

1. Do you remember studying classroom English sentences using an audio CD?

2. What is your feedback?

The results of the first question are shown in Figure 6.

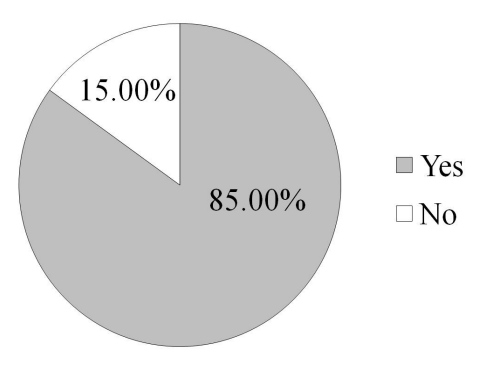

Figure 6. Do you remember studying classroom English sentences using an audio CD?

The results revealed that 17 of $20(85 \%)$ students had answered "Yes," which means that they remembered using a TTS audio CD when they had learned classroom English by RA instruction in June 2009, 2.5 years ago. The results of our study could also support the importance of listening and repeating practice in retaining what students had learned. 
Kataoka, H., Ito, M., \& Yamane, S.

The students were asked to write their comments freely in the second item of the questionnaire. Their comments are summarized in Table 9.

\section{Table 9}

Students' Comments in the RA Instruction Group $(n=20)$

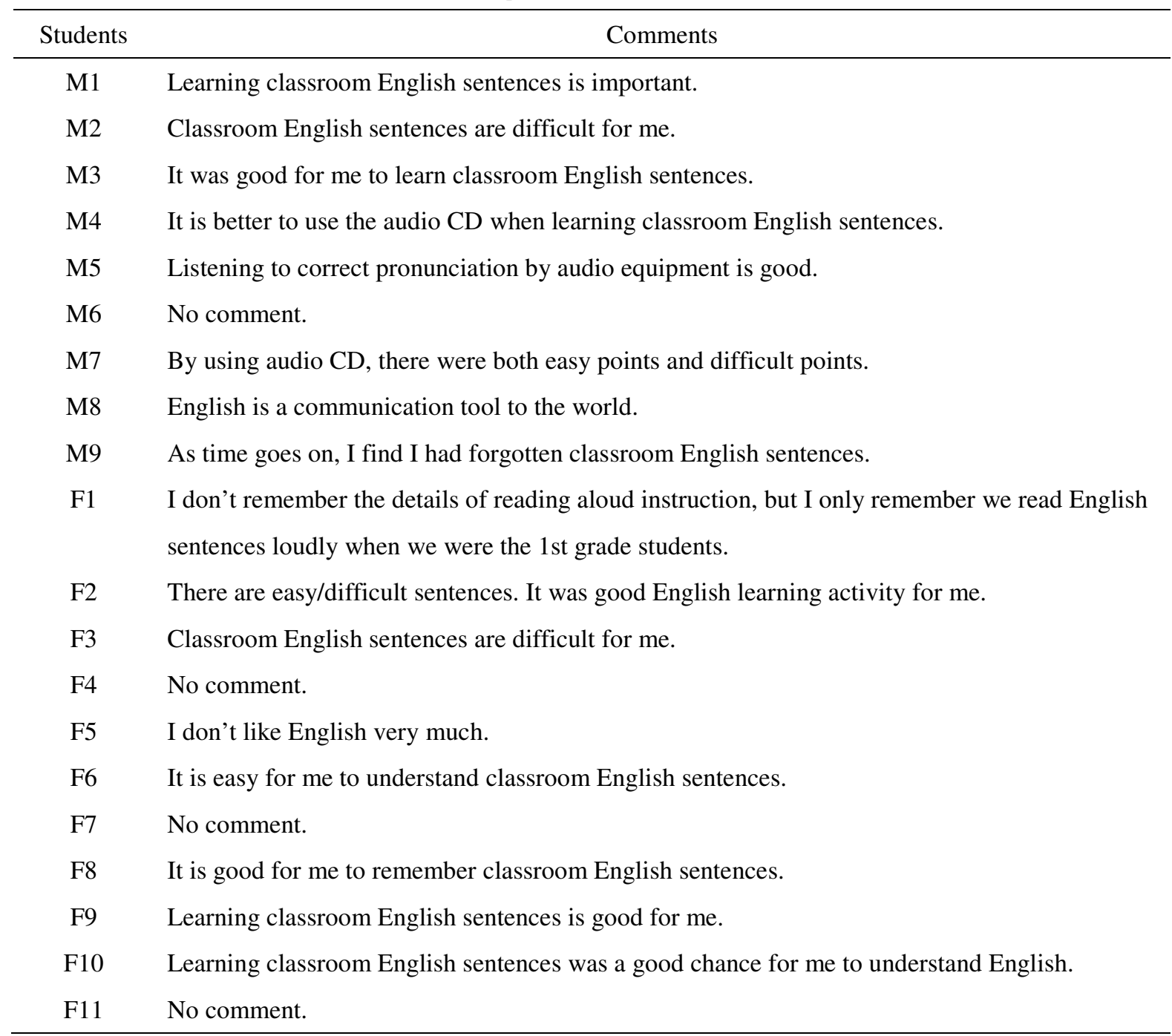

Note. "M" represents male students, and "F" represents female students. We translated the students' Japanese comments into English.

According to the students' comments (see Table 9), we found that many of them had a positive perspective in learning classroom English sentences. This suggests that an activity of RA classroom English sentences with audio could enhance students' learning motivation in a Japanese high school EFL setting.

\section{Summary and Concluding Remarks}

This study focused on the retention of classroom English sentences by RA instruction using TTS speech sounds for Japanese high school EFL learners. In order to compare the learning effect by an RA activity, two groups, an RA instruction group and a non-instruction group, participated in this longitudinal study.

The two Research Questions in this study were as follows:

1. How much difference, if any, in accurate retention rate exists between the RA instruction group and the non-instruction group?

2. What do the students in the RA instruction group think about the RA activity? 
Our findings revealed two sets of results.

First, concerning Research Question 1, the results obtained from the pre- and two post-cloze tests in the RA instruction group showed statistically significant differences after RA instruction, and the RA instruction group had a higher retention rate compared to the non-instruction group from June 2009 to December 2011 (see sections 6.1 and 6.2).

Second, concerning Research Question 2, the results show that $85 \%$ of the students remembered that they had learned classroom English sentences by RA instruction using a TTS CD (see Figure 6), and many of the students noted positive comments and feedback in learning classroom English sentences (see Table 9).

Both the RA instruction group and the non-instruction group in this study learned English by means of the high school English language learning curriculum specified by MEXT for Japanese EFL education. The difference in learning environment for these two groups was that only the RA instruction group had learned classroom English sentences during their five regular English lessons (see Table 3). Therefore, the results of both research questions revealed that although the RA instruction was provided for a short period, it yielded positive effects on the retention of classroom English learning from June 2009 to December 2011 (i.e., for 2.5 years).

Next, our study has the following two limitations:

1. We were unable to collect reliable data (e.g., how much time they had actually spent learning classroom English sentences by self-directed learning at home, although their teacher had instructed them to do so) from students in the non-instruction group.

2. The experiment period was from April 2009 to December 2011, and regular English lessons under the guideline of "the course of study in Japanese high school education" by the MEXT were given to all students in both groups. Considering the learning situation of participants in this longitudinal study, the regular English lessons could have influenced the posttest results.

Given these limitations, some of our conclusions remain tentative. To overcome these shortcomings, the research design should undergo further revisions (a) to collect accurate and reliable data from self-directed learning in the non-instruction group, and (b) to eliminate the influence from the regular English lessons.

English audio can be of great help for Japanese learners to study English (Hatori, 1977; Kohno, 1984; Palmer, 1921/1999). However, almost all of the audio language learning materials used in Japanese high schools are produced by commercial publication companies. We Japanese-English teachers sometimes feel that such audio materials do not fit our students' English proficiency. Unfortunately, some teachers think that using the TTS system would be difficult for them. They also have negative stance toward using TTS speech sounds, because TTS speech sounds are artificially synthesized speech sounds and there are not enough research results on how to use TTS speech sounds effectively for Japanese high school EFL education. These are the reasons why many teachers in Japanese high schools do not use the TTS.

However, using TTS speech sounds will be one way for teachers who want to improve audio materials suitable for their students, based on their English proficiency level. It is possible for teachers to use the TTS, because creating English audio is not very difficult (see section 3.3). By creating audio materials, teachers can provide their students with more opportunities of listening to English, thus can help enhance their English proficiency. The teachers can help develop their students' English proficiency little by little, by using TTS speech sounds during their lessons.

Finally, we hope that our findings in this study encourage more English teachers in high schools to use TTS speech sounds to create audio materials for RA instruction in their classrooms. The authors feel that additional studies on RA using TTS speech sounds should be conducted in Japanese high school EFL education in the future. 
Kataoka, H., Ito, M., \& Yamane, S.

Notes

1. Author 1 had bought TTS audio database CD in April 2007. It was $\$ 50$ including postage charges. She received permission through the selling agent of an audio database to create TTS CD-ROM for this study.

2. According to Pallant (2007, p. 6, p. 95), Cronbach's alpha of a scale should be above 0.70 .

3. A two-tailed independent $t$-test (Mizumoto and Takeuchi, 2008, p. 62; Mizumoto, 2009): Effect size $d=$ 0.20 , small effect size $(\mathrm{S}) ; d=0.50$, medium effect size $(\mathrm{M}) ; d=0.80$, large effect size $(\mathrm{L})$.

4. We received permission to conduct this research from the Prefectural Board of Education in Japanese Government which has jurisdiction over a public high school in this study.

\section{References}

Abe, F. K. (2001). Classroom English for better communication [Kyoushitsu hureai eigo hyougenshuu]. Tokyo: Pearson education.

Azuma, J. (2008). Applying TTS technology to foreign language teaching. In F. Zhang \& B. Barber (Eds.), Handbook of research on computer-enhanced language acquisition and learning (pp. 497-506). New York: Information Science Reference. http://dx.doi.org/10.4018/978-1-59904-895-6.ch029

Chew, L, C. (2009). Promises and challenges of e-assessments: A case of multimedia listening testing. LET Kansai Chapter Collected Papers, 12, 1-19.

Dutoit, T. (1997). An introduction to text-to-speech synthesis. Dordrecht: Kluwer Academic Publishers. http://dx.doi.org/10.1007/978-94-011-5730-8

Ebbinghaus, H. (1998). Memory: A contribution to experimental psychology [Über das gedächtnis: Untersuchungen zur experimentellen psychologie]. (H. A. Ruger, \& C. E. Bussenius. Trans.). Bristol: Thoemmes Press.

Halliday, M. A. K., \& Greaves, W. S. (2008). Intonation in the grammar of English. London: Equinox.

Hatori, H. (1977). Psychology of teaching English [Eigokyouiku no shinrigaku]. Tokyo: Taishuukanshoten.

Hirai, A., \& O'ki T. (2011). Comprehensibility and naturalness of Text-To-Speech synthetic materials for EFL listeners. JACET Journal, 53, 1-17. Retrieved from http://ci.nii.ac.jp/els/110008897622.pdf?id=ART0009855307\&type=pdf\&lang=jp\&host=cinii\&order_n $\mathrm{o}=\& \mathrm{ppv} \_$type $=0 \&$ lang_sw $=\&$ no $=1423737933 \& \mathrm{cp}=$

Hughes, G. S. (1981). A handbook of classroom English. New York: Oxford University Press.

Ishihara, Y. (2002). An empirical study on the effectiveness of reading aloud in English language reading classes at a senior high school level [Koukou Reading no jugyou niokeru ondoku no kouka ni tsuiteno ichikousatsu]. Hiroshima Daigaku Huzoku Cyuu-koutougakkou kiyou, 49, 71-75.

Jones, C., Berry, L., \& Stevens, C. (2007). Synthesized speech intelligibility and persuasion: Speech rate and non-native listeners. Computer Speech and Language, 21(4), 641-651. http://dx.doi.org/10.1016/j.csl.2007.03.001

Jones, D. (1976). An outline of English phonetics $\left(9^{\text {th }}\right.$ ed.). Cambridge: Cambridge University Press.

Kataoka, H. (2007). The study of memory: Retention of English and Japanese proverbs. Unpublished masteral thesis, Kansai University, Osaka.

Kataoka, H. (2009). The use of Text-To-Speech (TTS) synthesis technology for English education: Speech recognition of Japanese EFL learners [Text-To-Speech (TTS) synthesis technology o katsuyoushita eigo kyouikukyouzai no kaihatsu to nihonjin no onseininshiki]. Journal of Kansai University Graduate School of Foreign Language Education and Research, 7, 1-33.

Kataoka, H., \& Ito, M. (2013). A comparative study on reading aloud: Instructions by Text-To-Speech synthesis sounds and a high school Japanese English teacher. The JASEC Bulletin, 22(1), 39-54.

Kohno, M. (1984). Remodeling of English language lessons [Eigojugyou no kaizou]. Tokyo: Tokyoshoseki.

Kohno, M., Ikari, Y., Ishikawa, K, Kadota, S., Murata, J., \& Yamane, S. (Eds.). (2007). Explorations into the 
Retention of English sentences learned by reading aloud using Text-To-Speech (TTS) speech sounds

mechanism of language and cognition [Kotoba to ninchi no shikumi]. Tokyo: Sanseido.

Komatsu, M. (2011). Acoustic phonetics [Onkyou onseigaku]. In H. Joo, T. Fukumori, \& Y. Saito (Eds.), Dictionary of basic phonetic terms [Onseigaku kihon jiten] (pp. 115-122). Tokyo: Bensei Publishing Inc.

Ladefoged, P. (2003). Phonetic data analysis: An introduction to fieldwork and instrumental techniques. Oxford: Blackwell Publishing.

Ministry of Education, Culture, Sports, Science and Technology. (2009). The course of study for senior high schools [Koutougakkou gakushuu shidou youryou]. Retrieved from http://www.mext.go.jp/component/a_menu/education/micro_detail/_icsFiles/afieldfile/2010/01/29/128 2000_9.pdf

Ministry of Education, Culture, Sports, Science and Technology. (2010). ICT practical use in course instruction [Kyouka shidouniokeru ICT katsuyou]. Retrieved from http://www.mext.go.jp/a_menu/shotou/zyouhou/1259413.htm

Miyasako, N. (2002). Does oral reading ability have a relationship with English language ability for senior high school students? [Koukousei no ondoku to eigoryoku wa kankei ga aruka]. STEP Bulletin, 14, 14-25. Retrieved from http://media.eiken.or.jp/association/bulletin/vol_14.pdf

Mizumoto, A. (2009). Stats Stuff. Retrieved from http://www.mizumot.com/stats.html

Mizumoto, A., \& Takeuchi, O. (2008). Basics and considerations for reporting effect sizes in research papers [Kenkyuuronbun ni okeru koukaryou no houkoku no tameni]. Studies in English Language Teaching, 31, 57-66.

Pallant, J. (2007). SPSS survival manual: A step by step guide to data analysis using SPSS for Windows ( $3^{\text {rd }}$ ed.). Maidenhead: Open University Press.

Palmer, H. E. (1999). The principles of language study. In the Institute for Research in Language Teaching (Ed.), The selected writings of Harold E. Palmer: Pāmā senshū (vol. 1, pp. 331-520). Tokyo: Hon-no Tomosha.

Someya, M. (1993). Classroom English expression encyclopedia [Kyoushitsu eigo hyougen jiten]. Tokyo: Taishuukanshoten.

Suzuki, J. (1998). A reappraisal of reading aloud: An empirical study on the effectiveness of reading aloud [Ondokusidousaihyouka: Ondokushidou no kouka ni kansuru jissyoutekikenkyu]. LET Kansai Chapter Collected Papers, 7, 14-28.

Takeuchi, O. (2003). Searching for better language learning strategies: Studies on good language learners in the Japanese FL context [Yoriyoi gaikokugo gakushuuhou o motomete]. Tokyo: Shohakusha.

Takeuchi, O., Ikeda, M., \& Mizumoto, A. (2012). Reading aloud activity in L2 and cerebral activation. RELC Journal, 43, 151-167. http://dx.doi.org/10.1177/0033688212450496

Tamagawa Academy and University. (2014). About Tamagawa [Tamagawa Gakuen ni tsuite]. Retrieved from http://www.tamagawa.jp/introduction/enkaku/history/detail_6081.html

Taylor, P. (2009). Text-to-speech synthesis. New York: Cambridge University Press. http://dx.doi.org/10.1017/CBO9780511816338

Tinkham, T. (1993). The effects of semantic clustering on the learning of second language vocabulary. System, 21(3), 371-380. http://dx.doi.org/10.1016/0346-251X(93)90027-E

Webb, S. (2007). The effects of repetition on vocabulary knowledge. Applied Linguistics, 28(1), 46-65. http://dx.doi.org/10.1093/applin/aml048

Widdowson, H. G. (1978). Teaching English as communication. New York: Oxford University Press.

Yoshida, S. (2008) Development and practice of an electronic phrasal verb wordbook with GIF animations. Paper presented at the Proceedings of the 3rd International Conference of World CALL 2008, 84. 


\section{Appendix A: Twenty Classroom English Sentences}

1. Who is absent today?

2. What's the matter with Tanaka-kun?

3. I'm sorry to be late.

4. May I go to the restroom?

5. Are you ready?

6. Can you hear me?

7. Raise your hands.

8. Sit straight.

9. Open your books to page five.

10. Make pairs.

11. Look at me.

12. I don't have my textbook today.

13. Time is up. Please stop writing.

14. Fill in the following blanks.

15. Take it easy.

16. You can do it.

17. Guess what?

18. In English, please.

19. Listen to me.

20. Have a good weekend.

Appendix B: Activity Sheet (1)

\begin{tabular}{|c|c|}
\hline & Classroom English Activity Sheet (1) \\
\hline 1. & $\begin{array}{l}\text { 先生 : 今日は誰が欠席していますか。 } \\
\text { Who is absent today? }\end{array}$ \\
\hline 2. & $\begin{array}{l}\text { 先生 : 田中君はどうしたの?（田中君が欠席していて、先生が他の生徒に尋 } \\
\text { ねるとき） } \\
\text { What's the matter with Tanaka-kun? }\end{array}$ \\
\hline & $\begin{array}{l}\text { 生徒 : 遅刻してしまって、すみません。 } \\
\text { Im sm sorry to be late. }\end{array}$ \\
\hline & $\begin{array}{l}\text { 生掼 : トイレに行ってきてもいいですか。 } \\
\text { May I go to the restroom? }\end{array}$ \\
\hline & $\begin{array}{l}\text { 先生 : みなさん、用意ができましたか。 } \\
\text { Are you ready? }\end{array}$ \\
\hline
\end{tabular}




\section{Appendix C: A list of Twenty Classroom English}

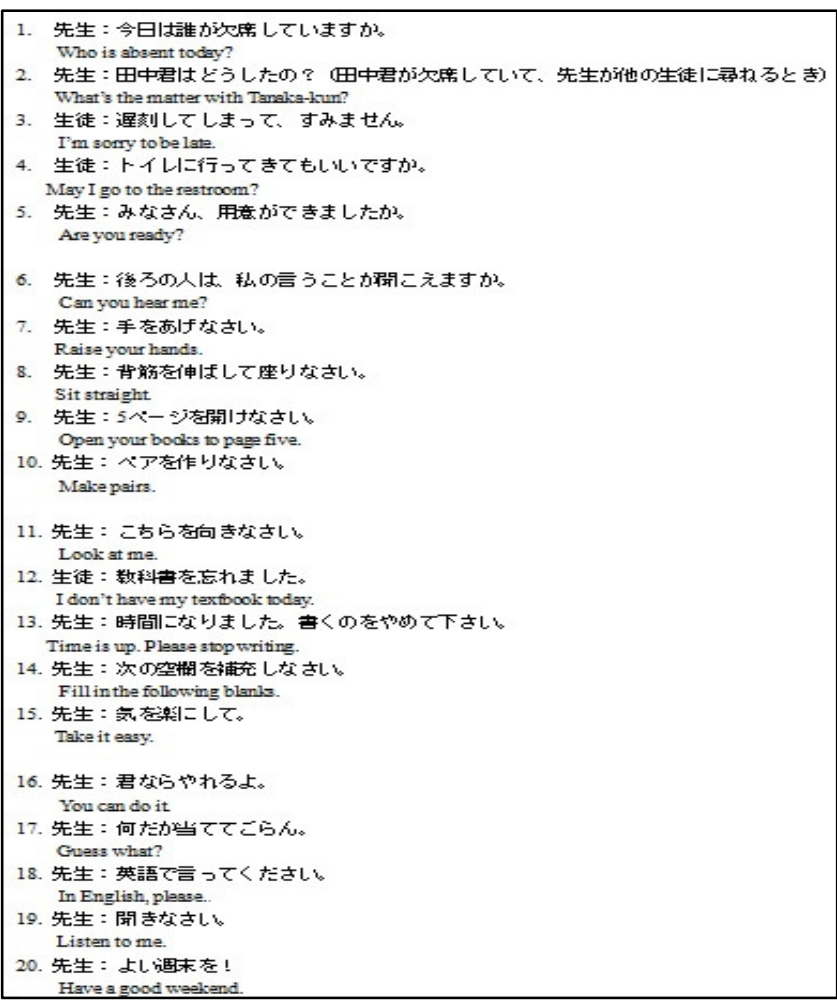

Appendix D: Cloze Test

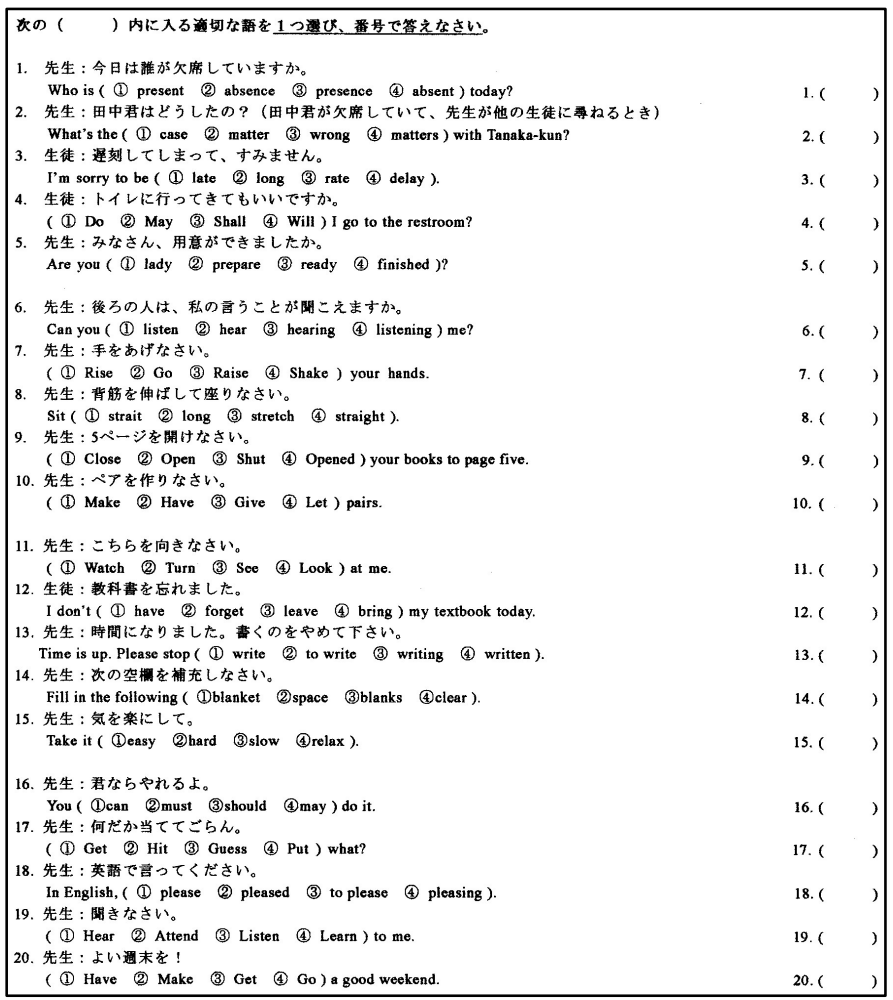


Kataoka, H., Ito, M., \& Yamane, S. 